\title{
Extériorité et objectivité du religieux
}

Un point de vue réaliste

Jean-Noël Ferrié

\section{OpenEdition}

\section{Journals}

Édition électronique

URL : http://journals.openedition.org/ress/2703

DOI : $10.4000 /$ ress. 2703

ISSN : $1663-4446$

Éditeur

Librairie Droz

Édition imprimée

Date de publication : 6 mai 2014

Pagination : 163-192

ISBN : 978-2-600-01829-6

ISSN : 0048-8046

\section{Référence électronique}

Jean-Noël Ferrié, «Extériorité et objectivité du religieux», Revue européenne des sciences sociales [En

ligne], 52-1 | 2014, mis en ligne le 12 mai 2014, consulté le 02 mai 2019. URL : http:// journals.openedition.org/ress/2703 ; DOI : 10.4000/ress.2703 


\title{
EXTÉRIORITÉ ET OBJECTIVITÉ DU RELIGIEUX \\ UN POINT DE VUE RÉALISTE
}

JEAN-NOËL FERRIÉ

CNRS / Centre Jacques Berque (Rabat)

jeannoel.ferrie@cjb.ma

Résumé. L'article soutient que le religieux ne consiste pas seulement en des croyances subjectives ou en des états mentaux, mais possède une objectivité indépendante des acteurs. II montre qu'une lecture perspicace de Durkheim peut aboutir à ce constat. Cette objectivité, toutefois, ne se limite pas à l'objectivité des formes symboliques produites par la culture. Elle est de nature dispositionnelle. De ce point de vue, on ne peut réduire l'attitude religieuse aux contenus précis des croyances.

Mots-clés: Durkheim, Lévi-Strauss, naturalisme, réalisme, religion, Wittgenstein.

\begin{abstract}
The article argues that religion is not merely subjective beliefs or mental states, but has objectivity independent from the actors. It shows that a perspicuous reading of Durkheim can lead to this conclusion. This objectivity, however, is not limited to the objectivity of symbolic forms produced by the culture. It is a disposition. From this point of view, we cannot reduce the religious attitude to the specific content of beliefs.
\end{abstract}

Keywords: Durkheim, Lévi-Strauss, naturalism, realism, religion, Wittgenstein. 
Dans l'étude qu'il consacre à Louis de Bonald, Koyré cite l'un des rares bons mots de celui-ci, selon lequel un déiste serait quelqu'un qui, durant sa courte vie, n’aurait pas eu le temps de devenir athée (Koyré, 198I, p. 127). Le déisme ou la religion naturelle apparait ainsi comme une étape sur la route menant à la négation de Dieu. Cette conception est assez conforme à ce qui nous vient facilement à l'esprit, lorsque nous pensons au siècle des Lumières. Ce n'est probablement pas faux, mais la disparition du divin n'est qu'une conséquence possible de l'idée qu'il existerait une religion naturelle. On peut, en effet, prendre cette idée au pied de la lettre, comme signifiant qu'il est possible de concevoir les êtres divins indépendamment des contenus théologiques qui leur sont référés. Cette position pose sans doute un certain nombre de problèmes, mais elle présente un remarquable avantage: permettre de penser la relation aux êtres divins comme un phénomène à part entière et non plus telle qu'on la conçoit le plus souvent, c'est-à-dire comme la conséquence secondaire ou l'adjuvent d'un phénomène social distinct, que ce soit la normativité religieuse ou la morphologie sociale'. Je voudrais donc soutenir une conception réaliste des êtres divins, ce qui implique d’admettre qu'ils ne sont pas liés de façon rigide à des contenus théologiques ou à des traditions culturelles.

Deux raisons justifient ma démarche. La première provient de l'impératif qu'il faut sauver les phénomènes (Quéré, 2004). Or, le phénomène distinctif de la religion est que l'on y entretient une relation avec des êtres divins. De trop nombreuses analyses semblent l'avoir oublié. Cet oubli apparaît de manière presque caricaturale, lorsqu'il s'agit de la religion des musulmans, où on croirait n'avoir affaire qu'à des adorateurs de la norme coranique. Pourtant, pour beaucoup de chrétiens et de musulmans (ainsi que de croyants d'une autre religion), la relation avec Dieu est spontanément perçue comme une relation avec un être plutôt qu'avec une norme; elle n'est pas davantage perçue, de façon dérivée, comme une relation avec un être, sous la condition de respecter la norme qu'il imposerait (voir Ferrié, 2004). La deuxième raison est la nécessité de réduire l'embarras dans lequel nous placent le mot «religion» ou l'adjectif 
«religieux». Une tentative pour sortir de celui-ci consiste, bien sûr, à formuler une théorie de la religion. Beaucoup l'on fait de façon plus ou moins assurée. D’une manière générale, pourtant, ces théories laissent toujours trop de faits et de phénomènes de côté. On se trouve confronté à une sorte de lit de Procuste épistémologique : on tire trop sur certains arguments et on en minimise abusivement d'autres. C’est certainement la conséquence de la tendance fautive, pointée par Wittgenstein (1996 [1965], p. 35), à rechercher derrière un substantif quelque chose de strictement factuel qui lui corresponde. En fait, il n'est tout simplement pas raisonnable de tenter d'embrasser, dans la même perspective, le culte nationaliste de Jeanne d'Arc, une prière faite devant une statue de la Vierge ou la certitude que le Coran est incréé. Chacune de ces pratiques et de ces états mentaux sont certainement aussi religieux que les autres, mais aucun, toutefois, ne relève du même ordre de réalité. S'il est donc erroné de rechercher une cohérence intrinsèque entre des ordres de réalité distincts, il n'en découle pas pour autant qu'il faille ignorer les phénomènes d'ordre, tant pratiques que conceptuels, qui permettent leur agrégation. De ce point de vue, poser l'extériorité du divin par rapport aux contenus qui lui sont rattachés, nous place d'emblée dans la perspective où le religieux se constitue de manière extrinsèque, de sorte que la relation entre le divin et ses contenus peut être conçue comme une relation actualisable et, partant, contextuelle, puisque les actualisations sont nécessairement liées à des états de choses spécifiques. Le religieux se révèle ainsi comme n’étant pas un tout cohérent déployant un système d'ordre dans le monde. Concevoir le divin comme n'étant pas rigidement lié à des contenus, nous libère de la nécessité de formuler des théories (voir Tarot, 2008) pour sortir de l'embarras dans lequel nous placent les multiples usages de la référence à la religion et aux êtres divins. En somme, en comprenant mieux la relation aux êtres divins, on comprend mieux ce qu'impliquent ou ne sauraient impliquer les multiples références au religieux qui émaillent le cours de la vie quotidienne dans les sociétés humaines.

Le présent article ne prétend pas justifier pied à pied l'idée d'extériorité des êtres divins ni la démontrer à partir d'une étude empirique. Il entend seulement établir que nous avons de bonnes raisons de considérer que c'est bien le cas et que ceci ne peut que faciliter notre analyse des phénomènes religieux 
et de la relation aux êtres surnaturels en général. Il montrera, en premier lieu, comment le développement de la connaissance des lois de la nature a conduit l'anthropologie et la sociologie à mécomprendre et à surdéterminer la nature sociale du religieux au détriment de la consistance des êtres divins (I). Cette surdétermination a souvent été attribuée à Durkheim, mais c'est sur la foi d'une mauvaise compréhension de son œuvre. Celle-ci, à condition de s'écarter de la légende holiste qui s'attache à elle, accorde, en effet, une consistance plutôt robuste aux êtres divins (2). D’une manière générale, ce n’est pas donc faire un bon usage de Durkheim que de considérer son œuvre comme fondant et justifiant une réduction sommaire du divin à la société. De fait, dans Les Formes élémentaires de la vie religieuse, il ne s'intéresse pas tant à la religion quau fonctionnement de l'esprit humain. Dans une perspective prima facie concordante, les approches naturalistes ont établi de manière convaincante que l'on ne pouvait réduire l'ensemble des comportements humains au social et à la culture. Il en va ainsi du religieux et, plus spécifiquement, de la relation aux êtres divins (3). Toutefois, le cognitivisme propose une conception trop réductionniste du religieux, qui ne tient pas compte du fait que, si les êtres divins sont extérieurs à la société, ils ne s'actualisent que dans le langage et que celui-ci est intrinsèquement lié à ce que Wittgenstein nomme une forme de vie. Le réductionnisme radical nous éloigne donc du phénomène visé (4). Il commet une erreur épistémologique, en faisant comme si les dispositions pourvoyaient à elles seules à l'existence des choses, alors qu'elles n'en sont que le ferment. En liant expressément, à la suite de Wittgenstein et de Lévi-Strauss, les dispositions à leur expression, c'est-à-dire aux formes symboliques et aux cadres sociaux, on ne bascule donc pas dans un monde de représentations. Les êtres divins ne sont, en effet, ni des pensées, ni des fictions culturelles, ni des constructions théologiques, ni davantage des êtres de plein exercice, comme on pourrait être tenté de le soutenir, pour leur rendre justice, en s'inspirant des travaux de Latour. Ils existent selon un mode d'être qui leur est propre, qu'il faut prendre pour ce qu'il est, mais qui ne déroge pas au réalisme et en fait ainsi des protagonistes de la vie humaine, que l'on soit croyant ou incroyant (5). 


\section{L'ÉLIMINATION DES ÊTRES DIVINS}

Dans son cours inaugural au Collège de France, en 1907, Jean Réville distinguait deux tendances dans ce qu'il considérait être l'étude «anthropologique » de la religion : la tendance psychologique, qui recherchait dans l'esprit humain la cause des faits religieux, et la tendance sociologique, qui la recherchait dans les phénomènes sociaux. La première était notamment illustrée par Tylor et la seconde par Durkheim (Réville, 1907, p. 208-209). Edward Tylor a défini la religion comme la relation avec les êtres spirituels (Tylor, I873, p. 424). Cette définition économique et robuste a été assez souvent reprise (voir Radin, 1957, p 9 ; Horton, 1960; Goody, 196I). Elle ne présente, toutefois, aucun engagement ontologique vis-à-vis des êtres divins : pour Tylor, ils sont le produit d'un dysfonctionnement cognitif consécutif à la difficulté dans laquelle se sont trouvés les hommes primitifs d'expliquer les rêves. En reformulant quelque peu la pratique distinction établie par Searle (1995), on dira que, pour Tylor, les êtres spirituels sont épistémologiquement vrais mais ontologiquement faux. On entend par là qu'il est factuellement vrai qu'ils sont vrais pour les gens mais faux qu'ils sont vrais indépendamment d'eux. Cette position n’est finalement pas très éloignée du classement opéré par L'Encyclopédie, qui d’un côté, fait aboutir la science de Dieu à la religion «d'où par abus, superstitions » et, de l'autre, à la science des esprits, aboutissant à la divination et à la magie noire (D’Alembert, 1984 [I75 I], p. 72). Le divin y est ainsi, non sans malice (Le Ru, 2006), assimilé à trois aberrations : la superstition, la divination et la magie noire. On connait, par ailleurs, la position de Hume pour qui la religion naturelle elle-même provenait de la superstition (Simon, 2009). L’idée commune à ces positions est que ce qui est ontologiquement faux ne peut exister épistémologiquement que sur la base d'une erreur de raisonnement ou d'une faiblesse de la pensée. Il n’est donc pas erroné de considérer qu’une partie des savants et des hommes instruits du XVIII et du XIX ${ }^{\mathrm{e}}$ siècles ont poursuivi une tâche d'épuration ontologique. Cette tâche s'est d'abord concentrée sur la nature du monde et les règles de son fonctionnement (Cassirer, 1990 [1932]). Comme il s'agissait de parvenir à un savoir assuré - scientifique, en un mot -, la question des croyances fautives, s'interposant entre la réalité et sa juste représentation, est devenue un 
thème majeur du développement de l'activité gnoséologique des Modernes. Le test ontologique appliqué à ces situations est celui de l'existence objectivable en dehors des croyances. La démonstration scientifique ou la comparution des entités visées étaient les deux moyens de cette objectivation. La forme de l'univers relevait de la démonstration scientifique et la description des espèces naturelles de leur comparution. Dans la mesure où les êtres divins ne pouvaient comparaître - personne n’a jamais rencontré Jupiter ou Athéna et personne n’a entendu Dieu parler à autrui ${ }^{2}$-, leur existence devenait à son tour l'objet exclusif d'une démonstration scientifique. Il en découla quelques aberrations baroques comme les dissections du cerveau pratiquées par Swedenborg afin de prouver la présence physique de l'âme. En fait, ainsi que l'a souligné Boudon, le développement de la connaissance des lois de la nature a entraîné une transformation de ce qui était crédible : connaissant mieux le fonctionnement de la nature, on ne pouvait, désormais, plus admettre un certain type de manifestations du divin auparavant admises (Boudon, 20ı, p. 30). La même rationalité ordinaire, qui avait permis d'y croire, l'interdisait à présent. Le déisme apparaît ainsi être l'expression d'une croyance en Dieu refusant ce qui heurte la raison ordinaire. La conséquence, non moins ordinaire, de ce refus est manifeste dans le chapitre que Renan consacre aux miracles dans sa Vie de Jésus: il les explique conformément à l'ordre naturel des choses (Renan, I863). Jésus n'y est plus un être divin. Sans doute, cette affirmation provoque-t-elle un scandale, qui chasse momentanément Renan de sa chaire, mais elle s'inscrit dans un climat favorable à la tendance consistant à séparer le divin du surnaturel, comme en témoigne presque simultanément la controverse Broglie-Guéranger sur l'intervention directe de Dieu dans la christianisation de l'Empire romain (Cuchet, 2or2a). Broglie ${ }^{3}$, bien que catholique convaincu, ne la juge pas nécessaire pour en rendre compte ; Dom Guéranger, le lui reproche, mais la position de Broglie est très largement soutenue par la hiérarchie catholique elle-même.

2 Comme le remarque justement Wittgenstein, 2008 (1929-1948), p. 158.

3 II s'agit d'Albert de Broglie (182I-190I), auteur de L'Église et l'Empire romain au Ive siècle et non de son frère cadet, l'Abbé de Broglie. 
Si l'élimination des êtres divins et, dans le cas qui nous occupe, de Dieu n'est pas un projet partagé par tous, une conception stricte de ce qu'ils peuvent être ou faire s'établit dans la durée, fondée sur le rejet du surnaturel, aussi bien de la part des croyants que de celle des incroyants. Contrairement à ce que nous incline à penser l'histoire des idées, ce n'est certainement pas dès le XVIII ${ }^{\mathrm{e}}$ siècle que la raison commune a sérieusement rogné les ailes des anges. Félicitant D’Alembert pour l'envoi qu'il lui avait fait de son Discours préliminaires de l’Encyclopédie, Buffon remarquait: «C'est la quintessence des connaissances humaines, mais ce suc n'est pas fait pour tous les estomacs, et je crois que vous n'aurez d'abord que l'admiration des gens de beaucoup d'esprit, et qu'il faudra vous passer pour quelque temps du suffrage des autres» (Buffon, I751). Les révolutions dans la pensée suivent ainsi le rythme des révolutions scientifiques auxquelles elles sont liées: si elles changent la donne pour les savants et les personnes directement concernés, elles laissent le reste du monde, c'est-à-dire sa plus grande part, en l'état (Kuhn, 2008 [1962], p. I57 et suivantes; Sharrock, Read, 2002, p. 50-52). C'est donc dans la durée qu'une certaine conception du surnaturel s'estompe. Les sciences sociales naissantes s'inscrivent ainsi dans un vaste mouvement dont les conséquences se déploient lentement. Elles s'établissent, en miroir des sciences de la nature (Winch, 2009 [1958]), en faisant leur le test d'objectivité en dehors des croyances, de sorte que les vérités épistémologiques des individus ne sont jamais tenues pour impliquant une quelconque vérité ontologique. Elles sont, en revanche, perçues comme symptomatiques des dysfonctionnements cognitifs affectant telle ou telle population, qu'il s'agisse des hommes primitifs eux-mêmes ou des primitifs contemporains, puisque, pour l'évolutionnisme courant, les étapes de l'évolution humaine laissent des survivances tenaces répondant «aux besoins intellectuels et moraux d'une grande partie des hommes, même dans les sociétés les plus civilisées » (Frazer, 1923, p. 23). Une vingtaine d’année plus tard, en 1945, Radcliffe-Brown remarquait sommairement à propos de la religion: «Il est hors de doute que l'histoire des religions est en grande partie l'histoire de l'erreur et de l'illusion» (Radcliffe-Brown, I968 [1952], p. 230). Il s'inscrivait ainsi très directement dans la suite de Hume, comme si, de l'un à l'autre, il ne s'était écoulé que la durée qui va d’un jour à son lendemain. 
L'élimination du divin apparaît ainsi comme la conséquence directe de l’attitude consistant à ne tenir pour réel que ce qui existe indépendamment de la subjectivité et subit avec succès l'épreuve de la comparution ou celle de la démonstration. La réalité n'est alors composée qu’à partir de l'ontologie naturaliste (Descola, 2005). Il en découle que les vérités épistémologiques des gens ne sont prises en considération que s'il existe quelque chose qui les rende vraies dans cette ontologie, en d'autres termes des «vérificateurs». Ici, un vérificateur est donc la contrepartie ontologique d’une vérité épistémologique. Le moine bourru de Sganarelle ne possède pas de vérificateur dans l'ontologie naturaliste, c'est-à-dire qu'on ne peut pas produire le moine bourru en public comme on produirait une girafe, dans un pays où les girafes n'existent pas, afin d’attester de leur existence. Il en est de même pour Dieu, pour les fantômes, les farfadets et les saints intercesseurs. À proprement parler, ils n'existent pas. Pourtant, ils ont une existence pour les gens. C'est ce que j’ai nommé, en me référant à Searle, une vérité épistémologique, en ce sens que cette existence pour les gens relève de la factualité. Le raisonnement naturaliste ne peut pas faire que les vérités épistémologiques sans vérificateurs disparaissent. Les gens continuent à croire en Dieu et, s'ils ne craignent plus le moine bourru, d’autres êtres l'ont remplacé. Les épidémies d’apparition mariale ou, plus tard, d'extra-terrestres en témoignent. La seule façon de rendre compte de l'entêtante présence de ces êtres consiste alors à en faire des aberrations, c'est-à-dire à les rattacher, non plus à un vérificateur, mais à une perception erronée de la réalité, c'est-à-dire à des états mentaux. Cette solution est coûteuse et largement inopérante. Elle est coûteuse parce qu'elle aboutit à faire d'une bonne partie des humains, des idiots. Ce coût est, du reste, totalement prohibitif, car qu'il n'apporte, en contrepartie, aucune autre compréhension des phénomènes et dissuade de prendre connaissance de l'insertion des vérités épistémologiques contestées dans une trame sociale et psychologique participant à la production d'une vie quotidienne normale et, surtout, parfaitement raisonnable, comme le souligne notamment Boudon à la suite de Weber (Boudon, 200I). La solution est, de plus, totalement inopérante : le réductionnisme ontologique n’est jamais parvenu à ses fins pratiques (Latour, 199I). Son seul résultat est d’avoir entrainé les sciences sociales à expliquer le religieux 
en ignorant la consistance des êtres divins, c'est-à-dire à gommer une partie des faits. L'explication s'est donc décalée: puisqu'elle ne pouvait en aucune manière partir des êtres et qu'expliquer, dans une perspective naturaliste (et de sens commun), consiste malgré tout à relier des vérités épistémologiques à des vérités ontologiques, les savants, les philosophes puis les spécialistes de sciences sociales sont donc passés des êtres aux croyances se rapportant à eux et les ont expliquées par la société, son état d’avancement et ses institutions.

\section{CE N'EST PAS LA FAUTE DE DURKHEIM}

Cette surdétermination par le social a souvent été attribuée à Durkheim, mais c'est sur la foi d'une mauvaise compréhension de son œuvre. L’affirmation lapidaire d’Evans-Pritchard: «pour Durkheim, Dieu est la société » (1971 [1965], p. 77) ne peut que nous induire ne erreur5. Pour Durkheim, il ne fait aucun doute que l'objet du culte rendu par les croyants est bien un être divin et non une entité collective (Spiro, 1972, p. I30) et que ce qu'ils éprouvent «ne peut pas être purement illusoire» (Durkheim, I968 [1912], p. 596). L'assimilation pur et simple des êtres divins à la société apparaît ainsi, moins comme le point de vue de Durkheim sur la religion, que comme celui que l'on pouvait se faire de son œuvre, en se plaçant dans la tradition éliminativiste que je viens de décrire. Mais, si l'on se place du point de vue revendiqué postérieurement à la parution des Formes élémentaires de la vie religieuse par Durkheim lui-même, il est clair que son but n'était pas - ainsi que l'a souligné Boudon (2000) - de produire une théorie sociomorphique du religieux mais d’avancer une théorie du fonctionnement de l'esprit humain dans son interaction avec le monde social (Durkheim, 1970 [1914] $)^{6}$. En ce sens, Les Formes élémentaires ne sont pas davantage, «tout simplement», une sociologie de la religion que La Pensée sauvage n'est, «tout simplement», une sociologie de la taxinomie des peuples sans écritures. Au demeurant, sur la question même de l'utilité sociale de la religion, Durkheim

4 Sur les erreurs d'interprétation auxquelles a donné lieu l'œuvre de Durkheim, voir Cuin, 201 I.

5 Sur la complexité du processus d'élaboration de la religion et du divin, voir Borlandi, 2012, notamment p. 374 et suivantes, ainsi que Paoletti, 2012a.

6 Sur cette position de Durkheim, voir notamment Paoletti, 2012b. 
apparaît assez éloigné des positions éliminativistes : il considère, en effet, qu’elle préserve l'ordre social en soustrayant certains domaines de l'action humaine au relativisme du libre arbitre. Il n'en fait donc nullement une aberration. De son point de vue, les vérités épistémologiques touchant à la religion n’ont pas besoin de vérificateurs impliquant des vérités ontologiques naturelles, car elles sont d'ordre pratique. Ce n'est pas Dieu qui rend vraie la prière mais le fait de prier?. Ce n’est pas Dieu qui rend vrai l’Église, c'est l'Église qui rend Dieu présent. En d’autres termes, c'est l'esprit humain qui produit l'ordre social. Il en découle que Durkheim prend la religion au sérieux, mais que ce n’est pas pour son contenu théologique.

Pourtant, la réception de Durkheim dans les milieux religieux s'est faite, au moins en un premier temps, sous le signe de la polémique et de la contradiction (Cuchet, 20I2b). Indépendamment même de la question de la vérité intrinsèque des ontologies divines, la priorité qu'il accorde aux rites et aux cultes sur les contenus théologiques ne pouvaient que déplaire aux croyants pour qui la religion est également - et parfois avant tout - un ensemble de contenus propositionnels. De leur point de vue, en effet, la question n'est pas seulement le culte mais le fait que leurs croyances puissent être considérées comme des vérités épistémologiques possédant des vérificateurs ou raisonnablement susceptibles d'en posséder. Mais cette divergence de point de vue - et c'est ici que je souhaite en venir - est, néanmoins, très différente de la divergence liée à la position éliminativiste, car elle porte sur les modalités d'être du divin et non plus sur sa présence ou son absence. Nous avons ainsi affaire à trois positions. Pour les éliminativistes, le divin est une illusion dont les conséquences sont néfastes. Pour Durkheim, il existe en tant que vérité épistémologique par les pratiques qu’il suscite et qui sont essentiellement bénéfiques. Pour les croyants, il existe en tant que vérité épistémologique ontologiquement fondée. On peut résumer (ceteris paribus) ces positions de la manière suivante : le divin n'existe pas, le divin existe dans notre esprit, le divin existe. La position

7 On retrouve ici le point de vue de Wittgenstein sur le rite. Ce n'est pas le contenu propositionnel de la croyance, qui lui serait associé, qui l'explique mais sa pratique elle-même (Wittgenstein, 1990 [1982]). Toutefois la position de Wittgenstein n'est pas complète (Le Du, 20II, § I3), puisque l'idée de prière est malgré tout grammaticalement indissociable de l'idée d'une entité à même de l'exaucer. 
de Durkheim est incomplète, car, si nous n'avons pas de problème à concevoir le monde sans Dieu ou avec Dieu - puisque dans les deux cas, c'est le monde tel qu'il est qui en détient la preuve -, nous avons besoin d'expliquer comment se manifeste cette disposition de l'esprit. Nous disons alors qu'elle se manifeste par des pratiques, et c'est là qu'il faut dire de quelles pratiques il s'agit et ce qui fait qu'elles paraissent appropriées. En d'autres termes, nous devons rendre compte d'un dispositif social. Durkheim a mis à notre disposition un puissant mécanisme explicatif répondant à cette nécessité : le sociomorphisme, dont l'utilisation a été différée dans les sciences sociales (tout au moins son utilisation subtile; voir Douglas, 1970) et qui permet de lier le style des relations entre les hommes et les êtres divins au style des relations que les hommes entretiennent entre eux dans une société donnée (Brown, I983, p. I2-16). Cette forme particulière d'isomorphisme donne une consistance spécifique aux êtres divins, puisqu'ils partagent ainsi des caractéristiques et des préférences que les hommes se reconnaissent communément, de sorte qu'ils ont acquis un important degré d’objectivité pour tout un chacun. Prier un saint ou Dieu, c'est, en effet, se placer dans un jeu de perspectives réciproques avec eux, puisque, indépendamment de tout ce qui nous sépare, nous supposons que nous pouvons concevoir de la même manière l'objet et la pertinence de la demande. De ce point de vue, les vérificateurs des vérités épistémologiques portant sur les êtres divins peuvent en venir à être aussi considérés comme d'ordre ontologique, mais ils s'agit d'ontologies sociales et non d'ontologies divines, en d'autres termes d'entités «institutionnelles » et non d'entités «brutes», c'està-dire indépendante de l’activité humaine, pour reprendre (en la transformant toujours quelque peu) une autre distinction de Searle (1995). Dieu, ainsi, n’est pas présent en tant que tel mais par le truchement d'un dispositif institutionnel plus ou moins développé. Nous ne nous éloignons guère de Durkheim, sauf que ce qu'il faut considérer, désormais, ce n’est pas la société d’un point de vue holiste et impersonnel - celle qui serait Dieu - mais des agencements sociaux à même de soutenir les caractéristiques des êtres divins. 
Une lecture de Durkheim dénuée de préjugés holistes peut donc nous conduire assez loin; au moins jusqu'à nous rendre compte que les ontologies divines sont soutenues par des dispositifs sociaux, de sorte qu'elles disposent d'une consistance objective (Ferrié, 20I3). Cette consistance est distincte des contenus théologiques, qui se rapportent aux êtres divins, puisqu'elle est obtenue par le réaménagement de dispositifs sociaux qui ne sont pas initialement religieux. C'est le sens fort du sociomorphisme. Par exemple, le culte des saints dans l'Antiquité tardive est dérivé des relations de patronage en usage dans la société romaine (Brown, 1983), ce n’est nullement une nécessité inhérente à la théologie chrétienne. On dira la même chose du culte des saints en islam, qui demeure - dans son idée même - controversé d'un point de vue théologique. Ainsi, Durkheim améliore-t-il (même si ce n’est pas son but) la position des êtres divins: de vérités dénuées de vérificateurs, c'est-à-dire de contreparties ontologiques, ils deviennent des vérités vérifiées par des ontologies, quand bien même s'agit-ils d'ontologies artefactuelles. Si nous revenons (toujours ceteris paribus) à nos trois positions par rapport au divin: (I) le divin n'existe pas, (2) le divin existe dans notre esprit et (3) le divin existe, nous pouvons, désormais, compléter la deuxième position qui devient: le divin existe dans notre esprit et s'actualise dans des dispositifs sociaux appropriés (2'). La position de Durkheim permet d'articuler une disposition de l'esprit et des contextes sociaux différents. Mais Durkheim n'est pas précis sur cette disposition.

\section{LE POINT DE VUE NATURALISTE SUR LES ÊTRES DIVINS}

Les travaux naturalistes sur le divin vont approfondir la connaissance de cette disposition, en s'attachant à préciser ce qu'implique, de leur point de vue, l'attribution de dispositions à l'esprit. Un point très important, souligné par Laurence Kaufmann (20II), dans sa défense du paradigme naturaliste, est la dissociation de la culture et du social et la bascule du social dans le domaine du développement naturel de l'espèce humaine. Cela veut dire qu'un certain nombre de nos conduites ne résultent pas du dispositif sémantique d'une culture localisée, mais de dispositions qui nous caractérisent dans la durée, indépendamment des sociétés considérées. À ce propos, l’anthropologie parlait «d’in- 
variants » et la psychologie classique de «nature humaine », dans une acception qui, bien que ne visant pas l'équipement cérébral, pointait néanmoins un état inhérent à l'homme dans son être spécifique. Car, si nous sommes enclos dans notre langage, comme le notaient aussi bien Wittgenstein (I993 [192I], p. 5-6.) que Lévi-Strauss (2008b [1962], p. 848-849), nous ne sommes pas, pour autant, une simple création de celui-ci, condamnés à n'être, à l'instar des personnages de roman, que des êtres de mots. On peut en tirer plusieurs conséquences. L’une d'elle est que nos dispositions précèdent la société dans laquelle nous vivons, ce qui peut aussi s'exprimer en soulignant que nous sommes toujours en décalage par rapport à ses dispositifs sémantiques. Cette attitude de décalage n’est pas une manifestation anomique mais un état normal de distance ${ }^{8}$.

Prenons un exemple. Dans une anecdote plusieurs fois reprises, RadcliffeBrown relate qu'un Européen, voyant un Chinois déposer un bol de riz sur une tombe, lui demande s'il croit que les morts mangent. Le Chinois répond à l'Européen en soulignant que sa question pourrait suggérer que les Européens déposent des fleurs sur les tombes, parce qu'ils pensent que les morts en apprécient leur parfum (Radcliffe-Brown, 1968 [1952]). La déférence vis-à-vis des morts est ainsi distincte des dispositifs sémantiques par laquelle elle s'exprime. Si l'on considère qu'il s'agit d'un invariant, les rites de l'Européen et du Chinois reçoivent chacun une explication satisfaisante - l'expression de la déférence - qui ne disqualifie aucune des deux croyances, puisque la substance de la croyance ne réside pas dans la particularité du rite. Ce qui réside dans la particularité du rite, c'est son appropriation à un contexte, le choix du riz plutôt que celui des fleurs ou l'inverse. On ne peut imaginer un rite qui ne soit pas approprié à un contexte. En même temps, on peut comprendre sans trop d'effort que des rites différents s'orientent vers la même chose. Il en est de même pour la croyance en Dieu : l'orientation vers un interlocuteur divin est distincte des mots et des configurations culturelles par lesquelles elle s'exprime. En ce sens, elle est à la fois dispositionnelle et contextualisée; dispositionnelle parce qu'elle a trait à l'homme et contextualisée parce qu'elle ne peut s'exprimer qu'en utilisant les significations dont elle dispose 
ou qu'elle réélabore. Les culturalistes, au contraire, pensent que les significations sont par elles-mêmes dispositionnelles. Ils croient, par exemple, que la manière de dire les choses possède une influence directe sur ce que l'on fait ou que l'on s'abstient de faire. C'est une lecture, peut-être naïve de la loi de Sapir-Worth, mais relativement répandue (à moins que ce ne soit la loi de Sapir-Worth qui soit la sophistication d'une croyance naïve). D’un point de vue dispositionnel, ce sont, au contraire, les manières de dire qui découlent de notre propension à être ou à faire. Le grand intérêt de l'approche naturaliste consiste ainsi à prendre les dispositions au sérieux, en les séparant nettement des dispositifs sémantiques.

Cette séparation implique, toutefois, une contrainte importante, d'où provient, pour une large part, la réception mitigée du naturalisme. Pour que les dispositions puissent être conçues de manière indépendante des dispositifs sémantiques, il faut qu'elles soient vraies, non seulement d'un point de vue épistémologique, mais également d'un point de vue ontologique, c'est-à-dire quelles impliquent une contrepartie dans le monde naturel. S’agissant d'une propension à être ou à faire attribuée à l'homme en général, la machinerie cérébrale peut apparaître à plus d'un titre comme le meilleur fondement ontologique possible des conceptions dispositionnelles. Ce réductionnisme est conséquent mais aporétique9. J'en donnerai les raisons dans la section suivante, mais ce qui nous intéresse, pour l'instant, ce n'est pas l'aporie mais l'apport du naturalisme.

Ceci nous ramène par un autre chemin à la question du réductionnisme. Il est inévitable : une description aussi fouillée soit-elle est une réduction de la réalité et une analyse est une réduction des descriptions. On ne peut donc refuser le réductionnisme, sauf à s'interdire de parler des choses et des états du monde. Cependant, il n'en découle pas que l'on ne puisse y mettre des limites, de sorte que les controverses sur le réductionnisme ne portent in fine que sur ses modalités. Un anthropologue américain, Stewart Guthrie (1993), a soutenu que la religion relevait d’une attitude générale vis-à-vis de l'univers - et dont les expressions sont, dès lors, légitimement comparables - impliquant la médiation d'êtres divins avec lesquels nous entrons dans un jeu de perspec- 
tives réciproques ${ }^{10}$. Il parlait de l'anthropomorphisme. Cette possibilité impliquait un réductionnisme radical par rapport aux théologies accompagnant chacune des représentations du divin impliquées. Dans cette perspective, Armin Geertz et Guðmundur Markùsson ont défendu l'idée que le théisme était naturel mais que l'athéisme l'était autant (Geertz, Markùsson, 20ıo). Les mécanismes psychologiques expliquant cette attitude, tels que les entend la psychologie cognitive, ont donné lieu à de nombreuses discussions (voir Barrett, Keill, 1996; Barrett, 1998). Le réductionnisme a donc permis de montrer que la religion était d'abord une orientation de l'esprit humain dans sa relation avec le monde, consistant à attribuer de l'intentionnalité aux non-humains, lesquels disposent alors de passe-droits, puisqu'ils bénéficient simultanément de caractéristiques relevant d’ordres de réalité différents. Ceci aboutit à des élaborations très diverses. C'est cette équivalence robuste entre des élaborations extrêmement différentes et parfois antagoniques - que l'on songe, par exemple, à l'opposition entre monothéisme et polythéisme - que l'approche naturaliste permet d'attester. On pourrait, sans doute, objecter que le spectacle de la diversité religieuse suffisait à ce constat. C'est une erreur. Nous ne sommes précisément sensibles aux concordances émergeant de cette diversité que parce que les progrès et la diffusion de la connaissance nous ont habitués à ne plus considérer les croyances des autres du seul point de vue des nôtres ${ }^{11}$. L'approche naturaliste s'inscrit ainsi dans la suite de la critique des religions révélées et de leur empire qui a notamment caractérisé, nous l’avons vu, la pensée du XvIII ${ }^{e}$ siècle. De plus, l’approche naturaliste à l'avantage de ne pas produire une théorie discriminante de l'attitude consistant à prêter une intentionnalité aux non-humains. Elle ne fait pas de distinction entre une pensée «primitive», en la matière, et une pensée «moderne». En d’autres

10 J'utilise une formulation qui n'est pas celle de l'auteur, mais qui ne dénature pas le point fort de son ouvrage.

II Ceci nous renvoie à la position de Boudon, citée supra, sur les conséquences des progrès dans la connaissance des lois de la nature, qui influe directement sur la place que nous accordons aux explications surnaturelles. Les sciences sociales possèdent un effet semblable: leur diffusion à une conséquence sur la manière dont nous considérons la vie sociale. II va de soi que cela ne signifie pas que le résultat de cette diffusion est conforme aux conceptions des savants et à l'état réel de la science. 
termes, elle n'explique pas l'orientation vers l'intentionnalité non-humaine par un état de civilisation déficient ou par les survivances d'un état primitif.

Il s'agit d'une médication efficace et donc d'un réductionnisme utile, d’autant plus utile qu'il nous tire vers la neutralité axiologique, toujours difficile à respecter dès qu'on parle de religion. J'en donne un simple exemple pour finir. D'un point de vue théologique et de sens commun, le catholicisme est un monothéisme. C'est ainsi que l'on comparera volontiers celui-ci à d'autres monothéismes et que l'on parlera couramment, à ce propos, des «trois monothéismes ». Pourtant, si l'on met de côté le contenu théologique en se concentrant sur l'ontologie - c'est-à-dire si l'on applique un réductionnisme similaire à celui utilisé par les partisans de l'approche naturaliste -, on est en droit de parler de polythéisme: un dieu père, un dieu fils et une mère montée au ciel dans une apothéose dénommée assomption, cela fait en tout trois divinités. On peut aussi rajouter les saints, d'autant plus facilement qu'on leur prête une efficacité miraculeuse, c'est-à-dire un type d'actions caractéristiques des êtres divins. D’une manière générale, le polythéisme nous apparaît comme quelque chose d'étrange, d'irrationnel et, au mieux, de baroque. Pourtant, si l'on considérait le catholicisme du simple point de vue ontologique, on y trouverait suffisamment de traits communs avec le polythéisme pour mieux comprendre celui-ci, c'està-dire pour le ramener à quelque chose de connu. Ce qui nous empêche de le faire, c'est probablement une déférence excessive pour les contenus, de sorte que nous sous-estimons largement la parenté entre les religions les plus diverses.

\section{L'APORIE DU NATURALISME}

Pour autant, l'approche naturaliste aboutit à une aporie que ne résolvent pas, me semble-t-il, les résultats indéniablement intéressants de la psychologie cognitive et de l'étude clinique de la machinerie cérébrale. C'est ce que l'on pourrait nommer l'aporie de Swedenborg. Elle concerne la pensée naturaliste en son entier. Swedenborg pratiquait des dissections humaines, afin de trouver le point de coïncidence de l'âme avec le corps. Il ne trouva pas ce point, abandonna ses dissections et devint le mystique que l'on sait, familier des espaces supraterrestres. S'il ne trouva pas le point où l'âme coïncidait avec le corps, ce 
n'est pas parce que l'âme n'existait pas, mais parce qu'il commettait l'erreur conceptuelle consistant à observer un phénomène dans un ordre de réalité dont il ne relevait pas. En disséquant un corps, on ne trouve, en effet, que des choses corporelles, de sorte que, si l'âme eut coïncidé avec le corps, c'est qu'elle en aurait été une partie et donc en rien ce qu'il recherchait, c'est-à-dire une âme. C'est un peu comme essayer de comprendre une chorégraphie en mesurant l’intensité des contractions musculaires. Le sens n’est pas dans le phénomène physique, même si celui-ci en est un support nécessaire. Les partisans de l'approche cognitivistes pensent que l'on peut être de plus en plus précis dans la description des mécanismes cérébraux, et sans doute est-ce vrai. Mais le point où une infime impulsion électrique devient une idée n'est guère plus observable que le point où l'âme coïncide avec le corps, parce qu'on parle de deux ordres de réalité différents: une idée, dans son acception sémantique, n’est pas un processus biologique, quelle que soit l'étendue que nous avons de la connaissance de celui-ci. C'est la limite au deuxième projet réductionniste du naturalisme, consistant à réduire les processus cognitifs à des processus cérébraux.

Une réutilisation de l'expérience de la «chambre chinoise» de Searle (1983) devrait permettre de clarifier cette distinction. Initialement, cette expérience de pensée est destinée à montrer que l'intelligence artificielle n’implique pas la compréhension des éléments de sens qu’elle traite. Un homme est enfermé dans une chambre, il reçoit des informations écrites en chinois, un livre lui indique, pour chaque groupe de caractère reçu, les réponses qu'il doit fournir, c'est-à-dire le groupe de caractère qu'il doit renvoyer. L'opération n'implique pas que l'homme comprenne un mot de chinois et, pourtant, tout laisse à penser, à l'extérieur de la chambre, que celui qui fournit la réponse comprend ce qu'on lui demande. Searle souhaitait ainsi distinguer les performances de l'intelligence artificielle des capacités de la cognition humaine, la première ne connaissant pas le sens des éléments qu'elle traite contrairement à la seconde. On peut réaménager cette expérience afin de rendre explicite la nécessité d'une extériorité aux processus cognitifs, fussentils naturels. Admettons que la chambre chinoise ne soit plus l'équivalent d'une intelligence artificielle mais du cerveau humain, c’est-à-dire de mécanismes, non plus informatiques, mais biologiques. Celui-ci traiterait des mots de manière non 
computationnelle, c'est indéniable, mais il en traiterait, toutefois, ainsi que c'est le cas dans la chambre chinoise, en n'utilisant pas les propriétés sémantiques que nous leur connaissons de l'extérieur. Il n'utilise pas le mot «bleu» pour traiter de la couleur «bleu». Il associe le mot bleu à quelque chose qui lui est propre mais qui n'est ni un mot ni bleu. Si le mot bleu lui était propre pour traiter le bleu, il ne pourrait pas, par exemple, utiliser le mot «azzurro», bien qu'il veuille aussi dire «bleu». De plus, notre cerveau est dépendant des informations qu'on lui fournit ainsi que l'homme dans la chambre chinoise est dépendant du livre qu'on lui a donné, c'est-à-dire d'une documentation appropriée sur le sens externe des phrases et des mots qu'il traite. Les processus cérébraux apparaissent ici dans leur inévitable incomplétude: la grammaire qu'ils appliquent et le lexique qu'ils utilisent s'élaborent en dehors d'eux dans des formes de vie mouvantes. Sans doute cette élaboration se fait-elle avec leur aide, mais cela n'en fait pas un processus interne pour autant. Nous avons plutôt affaire à un processus inextricablement collaboratif, de sorte que si - pour reprendre l'expression de Ryle (2005 [1949]) - l'esprit est bien lié à la machine, il n'est pas dans la machine mais à l'extérieur de celle-ci ${ }^{12}$.

La première conséquence de ceci est que la sophistication de la connaissance des processus mentaux soutenant les ontologies divines, en d'autres termes le réductionnisme cognitiviste, nous fait perdre de vue une partie du mécanisme dont elles procèdent. En effet, ce n’est pas en expliquant par quelle chimie cérébrale les hommes envisagent des intentionnalités non-humaines dotées d'attributs divins que nous comprendrons mieux le sens qu'elles revêtent pour eux ni la manière dont ils personnalisent ces intentionnalités pour en faire des protagonistes crédibles. On retrouve, ici, l'idée d’un feuilletage hiérarchique de la réalité, donnant la priorité aux niveaux les plus profonds sur les niveaux superficiels. Or, à une certaine profondeur, les actions bifurquent irrémédiablement : les contractions du muscle cessent d'être de la danse et les mots cessent d'être des mots. La disposition consistant à doter les non-humains d'une intentionnalité n'est, en effet, connaissable que dans la mesure où elle s'exprime dans des croyances, c'est-à-dire dans choses publiques et partagées; au demeurant, c'est l'air de famille associant ces croyances, qui nous 
fait inférer l'existence de cette disposition. Pareillement, les enquêtes d'inspiration cognitiviste portant sur les ontologies (la croyance en l'existence de diverses catégories d'êtres plus ou moins aptes à la comparution ${ }^{13}$ ) impliquent nécessairement de poser des questions en utilisant des mots insérés dans une grammaire entendue au sens de Wittgenstein, c'est-à-dire le domaine de pertinence d'un mot tracé par l'usage (voir Grysman, Hudson, 20I2). Les recherches menées sur de jeunes enfants montrent combien leurs ontologies sont dépendantes des informations véhiculées par leur environnement (Harris et al., 2006). De plus, les cas eux-mêmes dont discutent les tenants de l’approche cognitiviste sont tirés de croyances présentes ou passées, en d'autres termes (et de leur point de vue) du niveau superficiel du feuilletage de la réalité. Ils nous parlent du Père Noël, de Zeus ou de Mickey (voir, par exemple, Barrett, 2008; Gervais et Henrich, 20I0). Mais pourrait-il en aller autrement? Dès lors que l'on enquête sur des phénomènes dont la manifestation est essentiellement sémantique, peut-on échapper aux échanges langagiers, c'est-à-dire à l'expression située des individus? Si personne ne dit: «je crois en Dieu», l'enquête sur la disposition à croire en Dieu est tout simplement dénuée d'objet ${ }^{14}$.

Je ne suis pas en train de soutenir que les travaux d'inspiration cognitiviste ne nous apprennent rien; ils nous apprennent, au contraire, bien des choses sur le fonctionnement de la cognition, mais ce qu'ils nous apprennent, ils nous l'apprennent en référence à des contextes langagiers et sociaux spécifiés et non parce qu'ils parviennent à relier de manière déterministe les vérités épistémologiques des gens à un soubassement cérébral. De fait, la seule matérialité attestable de la disposition à doter les non-humains d'intentionnalité et à sélectionner parmi eux des interlocuteurs spécifiquement divins réside dans ses actualisations. En d'autres termes, la seule matérialité de la disposition, c’est son expression. Ainsi, autant le premier réductionnisme des naturalistes nous est utile pour établir des comparaisons, autant le second nous égare-t-il dans la recherche d'un soubassement matériellement distinct de l'expression, car il ne peut l'être que d'un point de vue analytique, autrement dit abstrait.

\section{Voir supra, section I.}

14 Peter Winch (2009 [1958]) nous donne de bonnes raisons d'adopter ce point de vue. 


\section{FORMES SOCIALES ET FORMES SYMBOLIQUES}

Il ne nous faut donc pas rechercher la justification ontologique des vérités épistémologiques concernant les êtres divins dans la machinerie cérébrale. Celle-ci ne fournit que l'illusion d'un soubassement dans lequel les naturalistes exileraient volontiers les divinités. Elles ne seraient alors rien de plus qu'une production erronée mais contextuellement inévitable de la pensée, ce qu’elles étaient déjà pour Tylor. Nous nous retrouvons ainsi dans la deuxième des trois positions par rapport au divin: (2) le divin existe dans notre esprit ${ }^{15}$. A l'instar de Tylor, les cognitivistes s'avèrent rétifs à l'alternative que l'on peut tirer d'une lecture perspicace de la position durkheimienne : (2') le divin existe dans notre esprit et s'actualise dans des dispositifs sociaux appropriés. Pourtant, il donne à celle-ci une assise non négligeable en montrant le caractère dispositionnel de la croyance aux êtres divins, redéfinie comme la propension à attribuer de l'intentionnalité aux non-humains et (secondairement) à les doter de caractéristiques faisant défaut aux hommes ${ }^{16}$. Néanmoins, il néglige le fait que cette disposition ne peut s'ancrer que dans le langage, c'est-à-dire dans son expression même (2"). Il s'agit d'une formulation conforme à l'alternative durkheimienne (2'). Je voudrais, dans cette section aller plus loin et montrer qu'elle permet d'établir l'extériorité et la consistance du divin par rapport à la religion sur la base de son insertion dans le langage.

Lévi-Strauss, dans la suite de Durkheim, souligne que la religion est d'abord l'un des domaines d'expression de l'activité intellectuelle de l'homme, dont la structure est indépendante des choses religieuses elles-mêmes. Toutefois, il s'éloigne de celui-ci en soulignant les limites du sociocentrisme, selon lequel la mise en ordre du monde par la pensée serait déduite de l'organisation sociale (Lévi-Strauss, 2008a [1962], p. 540 et suiv.). Il considère, on le sait, que cette mise en ordre procède directement du fonctionnement de l'esprit humain, de sorte que les partitions et les institutions sociales en sont

15 Voir supra.

I6 Voir notamment Boyer (200I) qui les tient pour «contrintuitives», ainsi que le compte rendu favorable de Clément (2003) et ma critique (Ferrié, 2012). 
l'expression plutôt que le modèle. Nous retrouvons ainsi avec l'esprit humain des formes symboliques et des dispositifs sociaux. Dans ce jeu, l'esprit ne se montre jamais à nu - du reste, il ne le pourrait bien sûr pas -, mais s'exprime simultanément dans les formes sociales et dans les formes symboliques qui s'attestent réciproquement en démultipliant les homologies, autrement dit les variations sur une petite série de thèmes. Les formes de vie et les jeux de langage présentent une intéressante similitude avec ce dispositif. Il ne s'agit pas d'établir des proximités entre deux auteurs qui s'ignoraient. Wittgenstein ne pouvait connaître Lévi-Strauss et Lévi-Strauss, me semble-t-il, n’y fait jamais référence. Pourtant - à travers des différences considérables que je ne méconnais pas, mais que je propose de mettre entre parenthèses - l'un et l'autre se sont préoccupés de l'articulation entre notre esprit, les formes sociales et les formes sémantiques. La position de Wittgenstein, telle qu'on peut la concevoir, notamment à la lecture des Recherches philosophiques (2005 [1953]), est qu'il est impossible d'avoir un langage, des règles ou une grammaire en dehors d'une communauté ou, plus exactement, que le langage, les règles et la grammaire impliquent le holisme (Le $\mathrm{Du}, 20$ I, p. 520-52I). Le langage présuppose, en effet, un cadre social. Ce que nous voulons exprimer n'a de sens qu'à l'intérieur des contraintes posées par une grammaire commune et cette communauté de la grammaire n'est possible que parce qu'il existe un cadre social approprié. Ce que j'ai à l'esprit n'est exprimable - c'est-à-dire est possiblement doté de sens - qu'à cette condition. Il en découle que ce qui justifie l'usage d'un mot ou d'un énoncé comme «Dieu existe» ou «Athéna m’a parlé », c'est la pertinence du mot ou de l'énoncé par rapport à une grammaire appropriée à un cadre social. «Athéna m’a parlé» ne peut avoir le même sens aujourd’hui que chez les anciens Grecs, même si lexicalement les mots disent la même chose. Ainsi, ce qui est commun à Durkheim, à Lévi-Strauss et à Wittgenstein, c'est que la justification ontologique des vérités épistémologiques ne se trouve ni en nous ni dans la nature - dans les faits bruts dont parle Searle (1995) - mais dans quelque chose d'extérieur au deux, et que cette extériorité est irréductible, quand bien même s'articule-t-elle avec notre esprit. Ensuite, s'étagent les différences. Chez Durkheim, c'est l'organisation sociale qui constitue cette exté- 
riorité $^{17}$. Chez Lévi-Strauss, elle est constituée par l’attestation réciproque que s'accordent les formes sociales et les formes symboliques. Chez Wittgenstein, cette extériorité est celle de la grammaire appropriée à des cadres sociaux ou celle des jeux de langages reliées à des formes de vie.

Ce qui est significatif pour les individus s'inscrit donc dans une double réalité à même de comparaître : les formes sociales et les formes symboliques ${ }^{18}$. De plus, les unes et les autres se consolident mutuellement. Si nous revenons à l'idée de dispositions à reconnaitre des intentionnalités non-humaines dans le monde, nous avons donc affaire à une «réalisation» de ces dispositions, puisque celles-ci se manifestent dans l'extériorité et dans des formes à la fois tangibles et crédibles. Ce qui rend possible le culte d'un être divin, c'est donc à la fois sa pertinence par rapport à nos habitudes sociales - le sociomorphisme de Durkheim - et le crédit que l'on accorde aux énoncés le concernant, ce crédit étant lui-même une conséquence de l’inscription des énoncés dans des cadres sociaux définissant la pertinence des énoncés. Certes, ces êtres n’ont pas la possibilité de comparaître devant nous comme une personne rencontrée dans la rue ou un homme célèbre que l'on verrait en direct à la télévision, mais ils possèdent, néanmoins, une consistance faite des formes sociales et symboliques se rapportant à eux. Ces formes sont à même de comparaître. On peut les désigner. Le tombeau d'un saint, le Christ du maître-autel, la première Pietà de Michel-Ange concourent à établir la réalité des êtres divins qu'ils évoquent. Un point essentiel est que cette réalité est alors distincte du fait d'y croire entièrement, partiellement ou de ne pas y croire. Même un agnostique possède une idée suffisamment claire de Dieu pour avoir le sentiment de parler de «quelque chose» et non pas de rien, quand il en parle. Le problème qui se pose n'est donc pas celui de l'existence en tant qu'entité mais celui des attributs de cette existence. L'existence de ces entités est, en

17 Une précision: pour Durkheim, l'organisation sociale est clairement une expression de la manière dont notre esprit fonctionne. La différence avec Lévi-Strauss porte sur le fait que, chez Durkheim, les formes symboliques sont déduites de l'organisation sociale alors que chez Lévi-Strauss, l'organisation sociale et les formes symboliques sont simultanément l'expression de notre esprit. II n'y a pas de priorité de l'une sur l'autre.

I8 C'est par ce double pouvoir d'attestation que Lévi-Strauss (1958) explique l'efficacité magique. 
effet, assurée d'emblée par des formes sociales et symbolique qui possèdent suffisamment d'objectivité pour qu'elle soit dotées d'une consistance initiale. Les modalités d'établissement des entités sont donc indépendantes de la question même du «croire »; et elles sont moins liées à des contenus précis - des énoncés propositionnels que l'on pourrait accepter ou refuser, ce qui déterminerait à soi seul l'existence des entités - qu’à des mécanismes courant de production de la réalité épistémologique. Lévi-Strauss montre ainsi, à partir d'un exemple emprunté à Evans-Pritchard, que la divinité des jumeaux est le produit d'une activité classificatoire aboutissant à une série d’analogies qui concourent à donner de l'épaisseur à cette classification; ces opérations sont totalement indépendantes de l'adhésion que l'on peut avoir vis-à-vis de cette classification (Lévi-Strauss, 2008a [1962], p. 523-524). La classification est un fait; l'adhésion en est un autre. C'est en ce sens que, pour lui, l'étude de la religion ne saurait renvoyer à des mécanismes précis, en un mot proprement religieux, mais au fonctionnement général de la pensée, c'est-à-dire à son expression dans des formes communément acceptables et, le plus souvent, acceptées (ibid., p. 546-547).

Ceci nous suggère que les êtres divins existent selon des «modes d'être » différents de ceux des êtres appropriés à l'ontologie naturaliste, mais qu'ils ne sont pas pour autant dénués de consistance. Dans une veine parfaitement durkheimienne, on pourrait dire qu'ils ne dépendent pas du bon vouloir ou de la croyance de tout un chacun pour exister. On peut donc, sur cette base, envisager l'existence robuste - épistémologique et ontologique - des entités divines ou, plus largement, des non-humains sans les doter d'une intentionnalité propre, à l'instar de ce que font de plus en plus communément les latouriens (voir Houdart et Thiery, 2010; Debaise, 20I2). En effet, l'existence d'un être ne nécessite pas son autonomie organique calquée sur les caractéristiques admises des «êtres vivants». C'est aussi une erreur de catégorisation que de confondre l'«existence » et le «vivant». D'une certaine manière, l'attitude naturaliste est à ce point implicite à notre conception de l'objectivité des phénomènes impliquant l'existence que leur réalité est conditionnée à leur capacité à être «vivants». Nous opérons, ce faisant, une réduction du 
réalisme au naturalisme, qui n'a pas lieu d'être. Non sans ironie, il en découle que des adversaires du naturalisme, partisans du constructionnisme, comme le sont les latouriens, en viennent à prêter une subjectivité propre aux nonhumains - c'est-à-dire une propriété caractéristique de la quintessence du vivant -, afin de stabiliser leur existence et d'assurer qu'ils seront sérieusement pris en compte dans l'explication des phénomènes. Pourtant, le réalisme ne requiert de nous que de rendre justice à l'objectivité de leur consistance.

Les êtres divins s'avèrent ainsi simultanément extérieurs à la religion et insérés dans le langage. Cette extériorité ne signifie nullement que la religion ne concourt pas à les spécifier, mais - précisément - concourir à produire un effet est tout autre chose que le produire à soi seul. On peut ainsi soutenir la réalité de Dieu à partir de la philosophie analytique (Swinburne, 2009 [1996]) ou à partir des développements de la science (Barbour, 1997, 2002 ; Guessoum, 20II) ${ }^{19}$. Certes, il y a alors une interférence entre les jeux de langages techniques des sciences et les jeux de langages techniques de la théologie, mais ces interférences ne créent pas un jeu de langage commun. Elles participent de ce vaste mouvement de synchronisation des formes symboliques qui fait que les composantes du monde paraissent toujours plus ou moins compréhensibles (même si nous les comprenons de manière erronée). Ceci dit, les ontologies divines ne sont pas nécessairement soutenues par des jeux de langage techniques. Des jeux de langage ordinaires peuvent suffire à cela. Prier Dieu de m'épargner un mal où le remercier d'un bien qui m’échoie ne demande que quelques mots. On dira que la prière s'adresse à «Dieu » plutôt qu'à «Athéna » ou à «Zeus», mais on aura le plus grand mal à aller plus loin et à rattacher le «Dieu» de la demande à une tradition scripturaire précise. Est-ce un musulman ou un chrétien qui dit: «Dieu, fais que Gina m’aime »? Et, si c'est le cas, est-ce un catholique, un orthodoxe ou un protestant, et de quelle sorte? Les renseignements qu'on peut obtenir ne proviennent que de la demande elle-même et portent directement sur Dieu ; c'est le jeu de langage de la prière. On tient ainsi pour acquis que Dieu est bienveillant et comprend 
les affaires humaines, puisqu'on peut lui demander de l'aide. On suppose qu'il admet l'amour comme préoccupation légitime. Enfin, si l'auteur de la demande se prénomme Clélia plutôt que Fabrice ou que la prière est formulée de la manière suivante: «Dieu, fais que Gina aime la pauvre fille que je suis », on suppose que Dieu admet tout aussi bien les amours homosexuelles que les autres. À ma connaissance, les religions monothéistes (considérées, du moins, depuis les jeux de langages techniques des hommes de religion) ne disposent pas d'une conception de Dieu cohérente avec cette demande, mais il n'en découle pas que le Dieu de cette demande soit inconsistant. Sa consistance ne provient pas de sa conformité aux normes d'une tradition religieuse exclusive, mais de l'entrelacement des formes symboliques (incluant les jeux de langages apparentés) et des formes sociales, sous-jacentes au jeu de langage où il est évoqué, et qui en établissent la factualité. Ce n’est sans doute pas le Dieu des rigoristes ni même celui de tous les croyants qui ne sont pas rigoristes, mais ceci ne change rien à l'affaire; du reste, ce n'est pas forcément le Dieu d'un croyant, car «Dieu » - ainsi que j'espère l'avoir montré - est disponible dans le langage pour tout un chacun. Il peut donc n'être, de ce point de vue, qu'un marqueur d'intensité.

On remarquera, pour finir, que le fait que les êtres divins disposent d'une consistance indépendante des contenus théologiques favorise la pluralité des morales. Le commerce avec les divinités - précisément parce qu'il est considéré depuis une perspective réaliste, c'est-à-dire durkheimienne ${ }^{20}$ - n'est tout simplement pas assimilable à un simple commerce avec des normes. Confondre les deux, c’est aplatir inconsidérément le réel.

NdA. Je remercie vivement Baudouin Dupret, Dominique Guillo et Virginie Tournay pour leur aide lors de l'élaboration de ce texte. 


\section{BIBLIOGRAPHIE}

BARBOUR Ian, 1997, Religion and Science, San Francisco, Harper.

-, 2002, Nature, Human Nature and God, Minneapolis, Fortress Press.

BARRETT Justin, 1998, «Cognitive Constraints of Hindu Concept of the Divine», Journal for the Scientific Study of Religion, 37-4, p. 608-619.

-, 2008, «Why Santa Claus is not a God», Journal of Cognition and Culture, 8-1/2, p. $\mid 49-161$.

-, KEILL Frank, 1996, «Conceptualizing a Nonnatural Entity: Anthropomorphism in God Concepts, Cognitive Psychologie, 31-3, p. 219-247.

BORLANDI Massimo, 2012, «La méthode de Durkheim à l'épreuve des Formes élémentaires », L'Année sociologique, 62-2, p. 367-385.

BOUDON Raymond, 2000, «Émile Durkheim: l'explication des croyances religieuses», Études sur les sociologues classiques, t. II, Paris, PUF, p. 63-123.

-, 200I, «La rationalité du religieux selon Max Weber», L’Année sociologique, 5I-I, p. $9-50$.

-, 2010, «La rationalité ordinaire, colonne vertébrale des sciences sociales», L’Année sociologique, 60-I, p. 19-40.

BOYER Pascal, 200l, Et l'homme créa les dieux, Paris, Laffont.

BUFFON Georges (Leclerc de), 175I, «Lettre de Buffon à D'Alembert du 20 juin $175 \mid$ », Correspondance de Buffon, édition électronique, Lettre L43:

$<$ http://www.buffon.cnrs.fr/>.

CASSIRER Ernst, 1990 (1932), La Philosophie des Lumières, Paris, Fayard.

CLEMENT Fabrice, 2003, «Les dieux disséqués. Vers une science du religieux», Critique, 677, p. 747-762.

CUCHET Guillaume, 2012a, «Comment Dieu est-il acteur de l'histoire? Le débat Broglie-Guéranger sur le "naturalisme historique" », Revue des sciences philosophiques et théologiques, 96-I, p.33-55.

-, 2012b, «La réception catholique des Formes élémentaires de la vie religieuse (1912) d'Émile Durkheim», Archives de sciences sociales des religions, 159, p. 29-48.

CUIN Charles-Henri, 20II, Durkheim. Modernité d'un classique, Paris, Hermann. 
D'ALEMBERT, Jean Le Rond, 1984 (I75I), Discours préliminaire de l'Encyclopédie, Paris, Vrin.

DEBAISE Didier, 2012, «Qu'est-ce qu'une subjectivité non-humaine? L'héritage monadologique de B. Latour», Archives de philosophie, 75-4, p. 587-596.

DESCOLA Philippe, 2005, Par-delà nature et culture, Paris, Gallimard.

DESCOMBES Vincent, 1995, La Denrée mentale, Paris, Minuit.

DOUGLAS Mary, 1970, Natural Symbols. Explorations in Cosmology, Londres, Routledge.

DURKHEIM Émile, 1968 (1912), Les Formes élémentaires de la vie religieuse, Paris, PUF.

-, 1970 (19|4), «Le dualisme de la nature humaine et ses conditions sociales», in Émile Durkheim, La Science sociale et l'action, Paris, PUF, p. 314-332.

-, 1970, La Science sociale et l'action, Paris, PUF.

EVANS-PRITCHARD Edward, 197I (1965), La Religion des primitifs à travers les théories anthropologiques, Paris, Payot.

FERRIÉ Jean-Noël, 2004, La Religion de la vie quotidienne chez des Marocains musulmans. Rites, règles et routine, Paris, Khartala.

-, 2012, «À chacun son puzzle. Pour un pluralisme méthodologique serein », SociologieS: <http://sociologies.revues.org/4016>.

-, 2013, «Jésus parle à Don Camillo. Analyse praxéologique des manifestations divines dans Le Petit monde de Don Camillo», Réseaux, 4-180, p. 15-38.

FRAZER James, 1923, Sur Ernest Renan, Paris, Librairie orientaliste Paul Geuthner.

GEERTZ Armin, MARKUSSON Guðmundur, 2010, «Religion is natural, atheism is not: on why everybody is both right and wrong», Religion, 40-3, p. I52- 165.

GERVAIS Will, HENRICH Joseph, 20I0, «The Zeus Problem: Why

Representational Content Biases Cannot Explain Faith in God», Journal of Cognition and Culture, 10-3/4, p. 383-389.

GOODY Jack, 1961, «Religion and Ritual: The Definitional Problem», British Journal of Sociology, 12-2, p. 142-164.

GUESSOUM Nidhal, 20II, Islam's Quantum Question: Reconciling Muslim Tradition and Modern Science, Londres, Tauris. 
GRYSMAN Azriel, HUDSON Judith, 2012, «Agency Detection in God Concepts: Essential, Situational, and Individual Factors», Journal of Cognition and Culture, 12-1/2, p. 129-146.

GUTHRIE Stewart, 1995, Face in the Clouds: A New Theory of Religion, New York, Oxford University Press.

HARRIS Paul et al., 2006, «Germs and Angels: the Role of Testimony in Young Children's Ontology», Developmental Science, 9-1, p.76-96.

HOUDART Sophie, THIERY Olivier, 20I0, Humains, non humains. Comment repeupler les sciences sociales, Paris, La Découverte.

HORTON Robin, 1960, «A Definition of Religion and Its Uses», Journal of Royal Anthropological Institute, 90-2, p. 201-226.

KAUFMANN Laurence, CORDONIER Laurence, 20II, «Vers un naturalisme social. A la croisée des sciences sociales et des sciences cognitives», SociologieS: <http://sociologies.revues.org/3595>.

KOYRÉ Alexandre, 1988, Du monde clos à l'univers infini, Paris, Gallimard.

KUHN Thomas, 2008 (1962), La Structure des révolutions scientifiques, Paris, Flammarion, «Champs».

LE DU Michel, 20II, «Méthode compositive et ordre social», Archives de philosophie, 74-3, p.509-523.

LE RU Véronique, 2006, «De la science de Dieu à la superstition: un enchaînement de l'arbre encyclopédique qui donne à penser», Recherches sur Diderot et sur l'Encyclopédie, 40/4I, p. 67-76.

LÉVI-STRAUSS Claude, 1958, Anthropologie structurale, Paris, Plon.

-, 2008a (1962), Le Totémisme aujourd'hui, in Fuvres, Paris, Gallimard, La Bibliothèque de la Pléiade.

-, 2008b (1962), La Pensée sauvage, in Fuvres, Paris, Gallimard, La Bibliothèque de la Pléiade.

PAOLETTI Giovanni, 2002, «Durkheim et le problème de l'objectivité: une lecture des Formes élémentaires de la vie religieuse», Revue française de sociologie, 43-3, p. 437-459. 
-, 2012a, «Les deux tournants, ou la religion dans l'œuvre de Durkheim avant les Formes élémentaires », L’Année sociologique, 62-2, p. 289-31I.

-, 2012b, «Durkheim's "Dualism of Human Nature": Personal Identity and Social Links », Durkheimian Studies, 18-I, p.61-80.

PIETTE Albert, 1993, «Implication paradoxale, mode mineur et religiosités séculières », Archives de sciences sociales des religions, 81-I, p.63-78.

-, 2013, L'Origine des croyances, Paris, Berg.

QUÉRÉ Louis, 2004, «Pour une sociologie qui «sauve les phénomènes», Revue du MAUSS, «Une théorie sociologique générale est-elle pensable? », 24.

RADCLIFFE-BROWN Reginald, 1968 (1952), Structure et fonction dans la société primitive, Paris, Minuit, 1968.

RADIN Paul, 1937, Primitive Religion: Its Nature and Origin, New York, Viking Press. RENAN Ernest, 1863, Vie de Jésus, Paris, Michel Lévy Frères.

REVILLE Jean, 1907, Les Phases successives de I'histoire des religions, Paris, Leroux. RYLE Gilbert, 2005 (1949), La Notion d'esprit. Pour une critique des concepts mentaux, Paris, Payot.

SEARLE John, 1983, «Minds, Brains and Programs», Behavioral and Brain Sciences, 3-3, p. 417-424.

-, 1995, The Construction of Social Reality, Allen Lane, Penguin Press.

SHARROCK Wes, READ Rupert, 2002, Kuhn, Philosopher of Scientific Revolution, Londres, Polity.

SIMON Alexandre, 2009, «L'histoire naturelle de la religion et les dialogues sur la religion naturelle», Philosophique, 12, p.93-122: <http://philosophique.revues.org/|47>.

SPIRO Melford, 1972, «La religion: problèmes de définition et d'explication», in Essais d'anthropologie religieuse, R.E. Bradbury et al. (éds), Paris, Gallimard, p. $109-152$.

SWINBURNE Richard, 2009 (1996), Y a-t-il un Dieu?, Paris, Ithaque.

TAROT Camille, 2008, Le Symbolique et le Sacré. Théories de la religion, Paris, La Découverte. 
TYLOR Edward, 1873, Primitive culture. Researches into the Development in Mythology, Philosophy, Religion, Language, Art and Custom, vol.I, Londres, John Murray.

WINCH Peter, 2009 (1958), L'ldée d'une science sociale et sa relation à la philosophie, Paris, Gallimard.

WITTGENSTEIN Ludwig, 1990 (1982), Remarques sur le Rameau d'Or de Frazer, Lausanne, L'Âge d'Homme.

-, 1993 (1921), Tractatus logico-philosophicus, Paris, Gallimard.

-, 1996 (1965), Le Cahier bleu et le Cahier brun, Paris, Gallimard.

-, 2005 (1953), Recherches philosophiques, Paris, Gallimard.

-, 2008 (1929-1948) Fiches, Paris, Gallimard. 